\title{
La parole de l'eau : symboles marins et répression sexuelle dans Les fous de Bassan
}

\author{
AuRÉLIE ChEVANT \\ HARVARD UNIVERSITY
}

Résumé : Cet article explore la signification psychanalytique de mythes marins dans Les fous de Bassan d'Anne Hébert. Complétant les travaux d'Evelyne Letendre et d'Isabelle Boisclair, cette étude examine comment des symboles comme la méduse et la sirène expriment le manque du féminin qui condamne les personnages masculins à un désir inassouvi et une violence incontrôlable. De plus, des symboles comme la petite sirène et le cygne démontrent comment les personnages féminins expriment un désir de rencontre avec le masculin qui est annihilé par le système patriarcal.

Mots-clés : Sexualité, Eau, Psychanalyse, Méduse, Sirène.

La communauté de Griffin Creek, représentée dans Les fous de Bassan, sera à jamais changée par la disparition de deux jeunes cousines, Nora et Olivia Atkins, à l'été 1936. Cette communauté consanguine, reposant sur un système patriarcal légitimé par une pratique religieuse, affecte autant les personnages masculins que féminins. Comme I'ont analysé Evelyne Letendre (2007) et Isabelle Boisclair (2008), Anne Hébert dépeint une société patriarcale séparant les hommes, source de pouvoir et d'autorité, et les femmes, objets de soumission et de passivité. Selon Boisclair, ce roman fait donc « une critique du système de sexe/genre hétéronormatif - système articulant trois volets de l'identité : le sexe, le genre et la sexualité - qui gère les rapports entre les hommes et les femmes tels qu'il les a lui-même construits. » (Boisclair, 2008 : 15) Letendre affirme pour sa part que « [c]e clivage prive aussi bien les hommes que les femmes de la présence de l'Autre [...]. Ce désir de rencontre avec le masculin se fait sentir chez Nora et Olivia Atkins, tout comme le manque du féminin transparaît chez les personnages masculins ». (Letendre, $2007: 33$ ) 
À partir de l'examen psychanalytique des mythes et des symboles marins associés aux personnages principaux, cette étude souhaite compléter les travaux de Boisclair et de Letendre. Tout d'abord, j'examinerai la représentation de la sexualité chez les personnages masculins. Je démontrerai que l'association de leur sexualité à des mythes comme celui de la méduse ou de la sirène indique un manque du féminin, en particulier de la mère, et le désir inassouvi de celle-ci. Ce manque entraîne une aliénation des personnages masculins, représentée par les « fous » de Bassan, oiseaux menaçants, symboles de violence, d'intrusion et de folie destructrice. Ensuite, j'analyserai la représentation de la sexualité féminine. La gémellité des personnages féminins symbolise un désir sexuel qui se situe entre des instincts primaires et le besoin de (re)connaissance du masculin et du féminin. Le désir de rencontre du masculin se retrouve dans l'image du cygne, symbole d'hermaphrodisme et de fusion entre le masculin et le féminin. De plus, le rappel du conte de la petite sirène permet de relever un besoin de compréhension de la sexualité féminine, non seulement par l'union avec le masculin mais aussi par la libération du féminin.

\section{La sexualité masculine : désir et répression}

\section{Le poisson : poids de l'héritage chrétien}

Vivant en autarcie sur une terre reçue du gouvernement canadien, la communauté de Griffin Creek s'inscrit dans le lignage de quatre familles consanguines : les Jones, les Brown, les Atkins et les Macdonald. Le pasteur Nicolas Jones, première voix de ce récit, semble guider ce « peuple élu » qui vit dans une terre soi-disant établie par Dieu, comme au début de la Genèse:

Au commencement il n'y eut que cette terre de taïga, au bord de la mer, entre cap $\mathrm{Sec}$ et cap Sauvagine. Toutes les bêtes à fourrure et à plumes, à chair brune ou blanche, les oiseaux de mer et les poissons dans l'eau se multipliaient à l'infini. Et l'esprit de Dieu planait au-dessus des eaux. (Les fous de Bassan, 1982)

Même si un tel passage nous offre l'image d'un lieu fertile et créateur, il montre aussi que ce peuple se trouve dans une position d'opposition entre le masculin et le féminin. Le Cap «Sec», par la présence de l'adjectif masculin, illustre la stérilité et la sévérité des hommes. Le Cap «Sauvagine » associe le féminin à des oiseaux aquatiques sauvages, que le suffixe « ine » fait ressortir, comme un diminutif. La femme peut donc être perçue comme dominée par des instincts sauvages. Cette distinction anticipe les tensions entre les hommes et les femmes de Griffin Creek. Même si Griffin Creek cherche à être un lieu béni par Dieu, il est aussi bientôt décrit 
comme un endroit de perdition, de limitation, et de mort : « Les Jones, les Brown, les Atkins, les Macdonald. On peut lire leurs noms sur les pierres tombales du petit cimetière dominant la mer. » $\left(F B^{1}: 14\right)$ Contrairement à la première description de multiplication des espèces à l'infini, ce deuxième passage présente une communauté consanguine qui se limite à quatre familles dont les noms sont immédiatement associés à la mort et à un lieu restreint.

De plus, commençant par les paroles prophétiques du pasteur Nicolas Jones et l'ambition de celui-ci de construire une annexe au presbytère contenant une galerie d'ancêtres « afin d'affirmer la pérennité de [s]on sang » ( $F B: 14)$, le récit permet d'identifier rapidement cette communauté comme un patriarcat religieux fondé sur des concepts traditionnels de sexualité masculine et féminine. Ce patriarcat religieux est à l'image des systèmes que décrit Gloria Anzaldúa dans son essai Borderlands :

The female, by virtue of creating entities of flesh and blood in her stomach (she bleeds every month but does not die), by virtue of being in tune with nature's cycles, is feared. Because according to Christianity and most other major religions, woman is carnal, animal, and closer to the undivine, she must be protected. Protected from herself. Woman is the stranger, the other. She is man's recognized nightmarish pieces, his Shadow-Beast. The sight of her sends him into a frenzy of anger and fear². (Anzaldúa, 1987: 17)

La religion est justifiée comme une protection pour l'homme et la femme : d'un côté, I'homme se protège de la colère et de la peur engendrées par la vision du féminin en imposant la volonté religieuse ; d'un autre côté, la femme se protège d'elle-même, et de sa nature démoniaque et sauvage, en respectant cette volonté religieuse. Suivant des principes religieux similaires, les hommes de Griffin Creek (en particulier Nicolas Jones et Stevens Brown) semblent ainsi se protéger des femmes en concevant la sexualité comme un devoir de procréation.

1. FB pour Les fous de Bassan, 1982.

2. Ma traduction: La femme, capable de créer des entités de chair et de sang dans son ventre (elle saigne chaque mois mais ne meurt pas), et d'être en accord avec les cycles de la nature, est à craindre. Selon le christianisme et la plupart des religions, comme la femme est charnelle, animale, et proche du non-divin, elle doit être protégée. Protégée d'elle-même. La femme est l'étranger, l'autre. Elle est la somme des parties cauchemardesques de I'homme, sa Bête de l'Ombre. La vision de celle-ci provoque un accès de colère et de peur chez I'homme. 
Dès le début du roman, le pasteur Nicolas Jones associe sa profession religieuse à son héritage familial masculin ( $F B: 14$ ). Il exclut les femmes de cette galerie d'ancêtres et se concentre essentiellement sur les hommes. En fait, il refuse de représenter les femmes lui-même, mais il confie la tâche aux jumelles, les filles de John et Bea Brown, qu'il a prises à son service. Cette « paternité » de substitution est pour le pasteur une manière de jouer à Dieu et de façonner ces filles à son image. S'occupant d'elles depuis un certain temps, il « [I] es [a] maintenues, corps et âme, dans cet état malléable, sans tenir compte du temps qui passe. » (FB:17) Il les maintient dans un état physique dénué de tout signe de sexualité et d'identité sexuelle. Chez lui, « tout se lave et se savonne quotidiennement, comme s'il s'agissait d'effacer une tache sans cesse renaissante. » (FB: 17) À coup d'eau savonneuse, Nicolas Jones lave du péché cet espace que les jumelles essaient d'envahir avec des symboles de I'héritage féminin de Griffin Creek. Ce «plaisir de peindre » $(F B: 16)$ chez les jumelles et leurs $œ u v r e s$ ne sont que des actes de folie qui doivent être purifiés.

De plus, Nicolas Jones ne reconnaît les femmes que comme un outil de procréation et de continuation de la lignée masculine. Comme sa femme Irène est stérile et qu'elle n'arrive pas à le satisfaire, elle est comparée à « un poisson mort » : «sa vie froide de poisson, son œil de poisson, sous la paupière sans cils, son odeur poissonneuse lorsque je m'obstine à chercher entre ses cuisses l'enfant et le plaisir. » ( $F B$ : 23-24) Cette image du poisson peut correspondre au symbole chrétien de l'Ichtus, poisson baignant dans l'eau bénite, symbole de fidélité et de longévité de la foi. Ainsi, lorsque sa femme n'accomplit pas son devoir chrétien de procréation, elle va à l'encontre de l'Ichtus. Le devoir de procréation passe ici avant le plaisir et semble même être le déclencheur du plaisir.

Stevens Brown, le frère des jumelles et l'un des personnages centraux du drame, semble aussi incapable d'appréhender le comportement et la sexualité des femmes et ne se sent rassuré que par le pasteur - seul homme qui sache apparemment contrôler les femmes et imposer son autorité. Ainsi en témoigne la première lettre de Stevens adressée à son ami Michael Hotchkiss en 1936 :

Ce que je déteste le monde feutré des femmes, leurs revendications chuchotées entre elles, à longueur de journée, l'été surtout, lorsque la plupart des hommes sont en mer, ou dans les champs. Il n'y a que mon oncle Nicolas pour les calmer et leur faire entendre raison. Au nom de Dieu et de la loi de l'Église qui sait remettre les femmes à leur place. (FB:88) 
Pour Stevens, la religion n'a d'autre vertu que de faire taire les femmes et de les soumettre aux désirs masculins. En fait, Stevens utilise la religion pour masquer une peur de l'expression féminine - vocale ou sexuelle. Il reprend l'image religieuse de I'Ichtus en associant le poisson à un symbole de protection contre les femmes :

Tout au long du voyage, si j'ai parfois travaillé au coton, avec les nègres, j'ai aussi passé des journées entières au vent du large, sur les grèves, penché sur les tables de vidage. Avec un couteau bien effilé je leur faisais sauter les entrailles aux petits poissons, miroitant au soleil, glissant entre mes mains. [...] Le soir venu je puais tellement que les femmes étaient bien obligées de me laisser tranquille. Le poisson, c'est comme si on entrait en religion, ça protège. ( $F B: 58)$

Stevens se sert du poisson, symbole chrétien de fertilité pour éviter tout contact physique avec les femmes. Ce blocage du désir féminin est recherché et même rassurant. Stevens trouve ainsi plus de plaisir à faire sauter les entrailles des poissons qu'à susciter l'attention féminine. Il s'appuie sur la religion pour valider son agression et sa violence envers les femmes.

\section{La méduse et la sirène : désir inassouvi pour la mère et peur de la castration}

Comme on a pu le voir, les hommes de Griffin Creek ont donc du mal à accepter la sexualité et le caractère charnel des femmes. Cette peur du charnel est rattachée à la peur du corps. Gloria Anzaldúa explique que les religions catholiques et protestantes encouragent la méfiance envers le corps en enseignant que le corps est un animal ignorant et que I'intelligence se trouve seulement dans la tête (Anzaldúa, 1987: 37-38). Dans Les fous de Bassan, cette peur du rapport avec le corps et les désirs charnels, semble trouver son origine dans la relation des hommes aux figures maternelles. Cela concerne principalement le personnage central féminin qui est la grand-mère de tous: Felicity.

Felicity Jones est la mère du pasteur Nicolas Jones et la grand-mère de Stevens, de Perceval, des jumelles, de Nora et d'Olivia. On constate, dès le début, à travers les paroles du pasteur, que la présence de la mère dans l'héritage familial est évacuée. Elle est soumise à un mari qui la trompe constamment et, en dépit de cela, ne se plaint jamais : «Ni une larme ni un cri. Felicity Jones met au monde des fils et des filles, selon le bon vouloir de son mari. Ni scènes ni reproches. » (FB:34) Dans cette communauté patriarcale, les femmes sont des objets liés à la reproduction de l'espèce. Cependant, Felicity s'échappe au petit matin pour nager seule. Cet acte, représentant une forme de satisfaction et de liberté féminine, affecte le pasteur pendant son enfance : «Elle écarte les bras et les jambes en étoile. Elle règne sur 
la mer. Sa robe de chambre, à ramages marron et rouge, flotte autour d'elle. On dirait une méduse géante. » ( $F B: 35)$ La façon dont la mère écarte les jambes et les bras, de manière volontaire et satisfaisante, et prend possession de l'espace, est terrifiante pour le petit garçon. L'image de la méduse rappelle ici le mythe en psychanalyse selon lequel le petit garçon, découvrant les organes génitaux de sa mère, est impressionné par l'absence de pénis. Le sexe de la mère provoquerait une terreur de l'absence associée à la peur d'une castration (Freud, 1974 : 273-274). Le rapport à la mère est donc fait d'envie et de rejet. Ce rapport ambivalent à la mère est aussi perceptible dans les souvenirs du jour où, à l'âge de douze ans, Nicolas confie à Felicity sa vocation de pasteur : «Elle m'embrasse pour la première fois. Son visage salé comme l'embrun. Une larme sur sa joue. Le long cou de ma mère. Son col baleiné. Son corsage noir piqué d'épingles où je n'ose appuyer ma tête d'enfant. » ( $F B: 25)$ Le corsage piqué d'épingles rappelle encore la méduse qui menace le masculin. La description de la mère est faite sous la forme d'un blason, technique utilisée lorsque, d'après Ann Rosalind Jones, I'homme réduit sa vision du corps féminin et concentre toute son attention et ses désirs sur une partie-objet lui permettant de renier ou de bloquer la vision du «manque » féminin qui le terrifie (Jones, 2000 : 86). De plus, le pasteur récite les psaumes de David afin de dominer la mère/mer: « Je les récite, debout sur un rocher dominant la mer. Je m'adresse à l'eau, désirant parler plus fort qu'elle, la convaincre de ma force et de ma puissance. L'amadouer tout à fait. La charmer au plus profond d'elle-même. Éprouver ma voix sur la mer. » (FB: 25) Les psaumes expriment les sentiments humains de I'homme devant Dieu, ils sont une prière de louange et de supplication adressée à lui. Le pasteur les utilise ici pour dominer la mère/mer et imposer sa voix. Comme sa mère ne semble pas répondre à ses désirs et attentions, Nicolas Jones cherche à lui enlever toute liberté d'expression. Si I'on reconnaît, comme Gaston Bachelard, dans L'eau et les rêves, que « la liquidité est [...] le désir même du langage » (Bachelard, $1942: 251$ ), alors on peut dire que Nicolas Jones étouffe toute forme de communication des femmes en utilisant la religion et en contrôlant leur relation au paysage maritime.

Tout comme le pasteur, Stevens a une relation ambiguë avec sa mère. La mère de Stevens est représentée comme une femme avec une très mauvaise circulation sanguine et qui dégage une froideur corporelle constante : « Ce froid vient d'ailleurs, des profondeurs confuses de la naissance, du premier attouchement des mains glacées de ma mère sur mon corps d'enfant. » ( $F B: 86)$ Le ventre de la mère se transforme ici en une sorte d'abîme et d'obscurité impénétrable, autre mythe féminin évoqué par 
Hélène Cixous dans «Le rire de la Méduse »(Cixous, $1975: 47)$. De son enfance, Stevens se souvient du froid de la mère intouchable et aussi de l'absence du lait maternel. Selon lui, «[c]'est pas le lait tout cru qu'elle m'a donné, Beatrice ma mère, c'est la faim et la soif. Le désir. Je dois hurler aussi fort que Perceval pour que mon père me chasse, à coups de pied, de la chambre froide. » $(F B: 87)$ Ce désir inassouvi du sein de la mère, très freudien, marque le début du manque et de la peur du désir féminin chez Stevens. De plus, son père le tape constamment sur le derrière et sur la tête afin « d'extirper du corps de l'enfant la racine même de la puissance mauvaise ». ( $F B$ : 87) Agissant comme une Loi du Père lacanienne, le père impose la castration en éloignant Stevens tout d'abord de la mère, puis du deuxième élément féminin de convoitise, Olivia (FB : 206), et enfin, en le poussant à aller en Floride.

Le retour à Griffin Creek en 1936 replace donc Stevens dans ce manque et dans un désir d'homme-enfant insatiable. Dès son arrivée, il compare la mer de cette terre natale « à une bête, étendue sur le dos, follement vivante, agitée par le flux et le reflux de son sang énorme. » (FB: 60) La bestialité, la position sur le dos et la folie mentionnées ici sont des éléments qui apparaissent à d'autres moments dans le récit afin d'exprimer le désir de Nicolas pour sa mère, libre dans l'eau ( $F B: 35$ ), ou bien encore de formuler l'envie violente de Stevens pour le sexe d'Olivia, fille de la haute mer ( $F B$ : 248). Afin de pouvoir faire face à cette mer (mère) originelle, Stevens ressent le besoin de s'asseoir près du ruisseau Brown Stream qui porte son nom. Le ruisseau fait penser à un élément masculin, comme un flot de liquide séminal, qui illustre la puissance masculine de Stevens. Cependant, ce dernier remarque :

J'ai mesuré mon corps d'homme, de la tête aux pieds, et j'éprouvais quelque chose d'obscur, de très fort et d'irréfutable à l'intérieur de moi ; la présence intacte de mon corps d'enfant, avec ses joies, ses peines et ses peurs. J'étais comme une femme enceinte, au bord d'un chemin, qui reprend souffle, après avoir beaucoup marché, et qui est lourde de son fruit. (FB: 60-61)

Stevens mesure sa masculinité à cette force féminine effrayante, et va se retrouver prisonnier de son désir sous la forme d'un souvenir heureux et douloureux. À défaut d'oublier ce manque maternel initial, il va porter son attention sur des substituts de mère/mer. À son arrivée au village, il se tourne immédiatement vers sa cousine veuve Maureen. Ayant été rejeté par la mère/mer, il se tourne vers une figure du même âge que sa mère dont le prénom présente un lien avec l'univers marin ${ }^{3}$.

3. La correspondance homophonique entre Maureen et marine est évidente ici. 
Stevens cherche donc le retour dans le ventre maternel/marin, et, quand il ne peut y retourner avec Maureen, il cherche à retrouver son enfance en « possédant » Olivia, deuxième figure du manque féminin de son enfance. Mais Olivia a trop grandi et elle l'effraie par son aisance corporelle. Il la regarde nager :

Je ne l'ai pas reconnue tout de suite, je l'ai même prise pour son frère Patrick, car nager dans cette eau agitée, faire les mouvements qu'il faut, prendre la vague au moment où elle se forme, se laisser porter par elle, descendre dans son creux et recommencer, comme si on faisait partie de la pulsation de l'eau, son propre cœur accordé à l'énorme cœur marin en mouvement, relève d'une telle maîtrise de son corps que je n'en croyais pas ma cousine capable. Son frère Patrick qui aime commander lui a sans doute donné des leçons de natation. ( $F B: 96$ )

Alors que Stevens n'est pas capable de maîtriser son corps d'homme, Olivia semble à l'aise dans sa sexualité féminine bourgeonnante. Bachelard identifie la mer comme un milieu dynamique dans lequel la nage est une «lutte en soi ». Plus que quiconque, le nageur peut dire : le monde est ma volonté, le monde est ma provocation. C'est moi qui agite la mer. »(Bachelard, 1942 : 225 ; I'auteur souligne) Olivia, par sa maîtrise des vagues et de la pulsation de l'eau, affirme sa volonté sur le monde, et comme elle brouille de telle sorte les frontières de la dualité masculin-force/autorité et féminin-vulnérabilité/soumission, Stevens est obligé de la repositionner dans un schéma patriarcal :

Je tente de la prendre dans mes bras, ruisselante et glacée, tout essoufflée, elle se débat comme un poisson fraîchement pêché, ses cheveux mouillés me passent sur la face en longues lanières froides. Je lui chuchote des propos galants un peu bizarres où il est question d'une sirène aux pieds palmés, dénoncée par Nora. ( $F B: 97)$

Contrairement à Stevens, Olivia est en harmonie avec l'eau natale et le lien avec la mère/mer. Pour affirmer son désir masculin, Stevens est obligé de la comparer à un poisson et en particulier à une sirène. L'image de la sirène rappelle le symbole psychanalytique de la peur de la sirène-mère qui entraîne une perte de la subjectivité. Joyce McDougall met en évidence que, selon Freud et Lacan, la figure de la sirène-mère menace d'envelopper ou de dévorer l'enfant qui est protégé par la communication verbale (McDougall, 1995 : 82). La voix de la mère ranime donc des fantasmes de fusion, ayant pour conséquence la perte de l'identité subjective et sexuelle ${ }^{4}$. En voulant séduire Olivia avec des propos maladroits, Stevens exprime une peur de la non-individualisation, et avec sa voix et pensée masculines, il emprisonne

4. Traduction de I'analyse de Joyce McDougall dans Many Faces of Eros : «The mother's voice rekindles fantasies of the wish for fusion, with the consequent loss of both subjective - and sexual - identity ». (McDougall, $1995: 82$ ) 
Olivia dans un mythe misogyne qui condamne sa liberté et sa sexualité.

De plus, comme Olivia déclenche en lui des réminiscences de l'incapacité à satisfaire son désir pour la mère, Stevens l'associe également à la figure d'un abîme qui menace de le détruire. Après avoir tué sa cousine, Stevens raconte à Michael Hotchkiss en 1982 que « les cris d'Olivia dans tout ce vacarme, tu penses bien qu'ils tombent comme des gouttes d'eau dans la mer. L'abîme de la mer nous contient tous, nous possède tous et nous résorbe à mesure, dans son grand mouvement sonore. » ( $F B: 247$ ) Stevens confesse indirectement sa peur et sa grande incompréhension du féminin, de quelque chose qui a une voix propre, mais qu'il essaie de brouiller dans un moment de folie:

Le vrai problème c'est de l'immobiliser tout à fait. L'injurier en paix. L'appeler salope. La démasquer, elle, la fille trop belle et trop sage. À tant faire I'ange on... Lui faire avouer qu'elle est velue, sous sa culotte, comme une bête. Le défaut caché de sa belle personne solennelle, cette touffe noire et humide entre ses cuisses là où je fornique, comme chez les guidounes... $(F B: 248)$

Il n'y a aucune communication possible entre lui et les femmes ; la sexualité féminine est à craindre et la femme n'est pas un ange mais un animal féroce prêt à dévorer le sexe masculin.

\section{Les fous de Bassan et la tempête : folies du prédateur masculin}

Les personnages masculins se retrouvent ainsi frustrés dans leur sexualité, une frustration qui se transforme en violence, voire en folie totale. Cette violence se retrouve dans l'évocation d'une mer enragée, malmenée par la tempête, et d'une nuée d'oiseaux menaçants, plongeant à pic dans l'eau.

La frustration de Nicolas Jones est alors perceptible dans la présence des fous de Bassan. Dans son livre en 1982, le pasteur observe des oiseaux volant au-dessus de lui :

De grands oiseaux migrateurs, en formations serrées, passent au-dessus de Griffin Creek, projettent leur ombre noire sur le presbytère. J'entends des jappements lointains, toute une meute céleste qui s'éloigne dans la nuit. Vais-je à nouveau mettre le nez dans mon péché ? ( $F B: 24)$

Les oiseaux sont un symbole divin qui lui rappelle son péché de 1936 lorsqu'il a touché Nora et a abusé d'elle. Ce côté obscur de sa sexualité plane au-dessus de sa légitimité religieuse : les oiseaux représentent son désir destructeur, et son inconsistance et hypocrisie religieuses. Revenant sur les débuts de sa vocation de 
pasteur, il reconnaît son incapacité à utiliser les paroles de Dieu pour dompter la mer/mère : «Pour le moment je jette les paroles de David par-dessus les flots. On dirait le vent qui brise la crête des vagues en plumes folles, éparpillées. Seul le cri des oiseaux aquatiques touche l'eau d'aussi près. » ( $F B: 25)$ Encore une fois, la religion n'arrive pas à calmer son désir de subjuguer la femme et les oiseaux marins symbolisent une telle faiblesse.

De la même manière, le désir et la frustration de Stevens apparaissent dans la violence de la nature et, en particulier, dans celle du vent sur la mer. La première fois que Stevens essaie de parler à Olivia, il s'aventure discrètement chez elle lors d'une soirée venteuse. Il l'observe repasser « une chemise d'homme » à travers la moustiquaire de la porte où le vent s'engouffre : «Ouverte aux quatre vents, cette fille est ouverte aux quatre vents, je n'aurai qu'à paraître pour... » (FB : 77) Lorsqu'Olivia est en mer ou sur la plage, elle devient moins accessible car elle fait partie de cette nature qu'elle domine; mais lorsque celle-ci est dans l'espace patriarcal de la maison, condamnée aux travaux ménagers au service des hommes de sa famille, elle devient « ouverte » au désir sexuel des hommes. De plus, Stevens avoue s'être jeté dans la grande tempête, « le plus profondément possible, au cœur de son épicentre, semblable à un fou ». ( $F B: 102)$ Bachelard, en reprenant Balzac, précise que la représentation $d^{\prime}$ « une tempête extraordinaire est une tempête vue par un spectateur dans un état psychologique extraordinaire. » (Bachelard, 1942 : 232-233; I'auteur souligne) La tempête est ainsi vécue par Stevens comme « l'expression de [s]a vie, de [s]a violence la plus secrète. » (FB : 102) Il s'agit d'une violence doublée d'une forme de folie représentée par la présence des fous de Bassan qu'il observe planer au-dessus de lui et se laisser « tomber, tête première, comme une flèche à la verticale. Ne ferm[ant] [leurs] ailes qu'au moment de toucher l'eau, faisant gicler dans I'air un nuage d'écume. » (FB : 238) Selon André Brochu, « [I]a description comporte un schème de pénétration, et le giclement d'écume qui s'ensuit a des connotations sexuelles évidentes. » (Brochu, 2000 : 193) En effet, la pénétration brutale, comme une flèche verticale indiquant un phallus en érection, dévoile un désir sexuel de toute-puissance sur l'élément féminin que représente la mer. L'image du fou de Bassan symbolise donc d'un côté la violence sexuelle de Stevens et d'un autre côté la folie qui en découle lorsque ce désir n'est pas assouvi. 
La sexualité féminine : instincts et (re)connaissance

\section{Entre terre et mer : gémellité et jouissance du corps}

Dans l'espace restreint et contrôlé de la communauté de Griffin Creek, les hommes dominent et les femmes sont soumises. Evelyne Letendre résume parfaitement une telle séparation et hiérarchie entre les sexes :

Ce clivage prive aussi bien les hommes que les femmes de la présence de I'Autre ; et cette privation suscite le désir de se rapprocher de l'Autre, malgré les interdits. Ce désir de rencontre avec le masculin se fait sentir chez Nora et Olivia Atkins, tout comme le manque du féminin transparaît chez les personnages masculins. (Letendre, $2007: 33$ )

Comme nous l'avons vu précédemment, les personnages masculins souffrent du manque du féminin, ce qui va les pousser à se sentir frustrés et belliqueux dans leur approche de la sexualité. Les personnages féminins, quant à eux, sont poussés par un désir ambivalent de rencontre avec le masculin. Selon Claudine Fisher,

[les personnages féminins] doivent se reconnaître dans leur multiplicité et dans une même origine de femme, eau, source, terre et mer, et dans la jouissance de leur corps, dont elles ont été longtemps privées. Nora comme Olivia se retrouvent dans leur mère et grand-mère et rejettent, comme la nouvelle génération de femmes, les conseils de prudence des aïeules. (Fisher, 2001 : 73)

Les personnages féminins doivent non seulement reconnaître leur multiplicité dans le désir sexuel, mais elles doivent également ignorer les voix de leurs ancêtres féminins qui symbolisent la soumission à la loi du silence imposée par le patriarcat.

La multiplicité de l'identité féminine apparaît tout d'abord dans le motif de la gémellité. Les jumelles, les sœurs de Stevens, sont les premiers personnages féminins représentés dans l'univers patriarcal. Bien que le pasteur soit ravi de les avoir maintenues « corps et âme, dans cet état malléable » ( $F B: 17)$, et de les avoir modelées sans « une once de graisse, ni seins, ni hanches » ( $F B: 17)$, les jumelles, à n'importe quelle occasion, échappent au carcan patriarcal et expriment une jouissance corporelle. Sur le mur des ancêtres mâles du pasteur, elles dessinent des têtes de femmes et trois noms, Nora, Olivia, Irène : «Bousculant toute chronologie, s'inventant des grands-mères et des sœurs à foison, les jumelles découvrent le plaisir de peindre. Éclaboussées de couleurs de la tête aux pieds, elles s'extasient devant leurs œuvres. » (FB:16) Le pasteur ne peut pas accepter que ces corps qu'il a modelés puissent ressentir un plaisir et une extase qu'il ne contrôle pas. De plus, elles écrivent « [é]té193619361936» (FB : 19) sur le mur et, dès que le pasteur a le dos 
tourné, « les jumelles retrouvent leurs secrets de jumelles, des rires étouffés, des gloussements, des caresses furtives. » (FB: 19) Ainsi, elles peuvent encore exprimer leur rire. Ce rire rappelle le rire de la méduse chez Hélène Cixous, car les jumelles refusent le silence et envahissent le mur du pasteur pour lui montrer qu'il faut regarder « la méduse en face pour la voir : et elle n'est pas mortelle. Elle est belle et elle rit. » (Cixous, $1975: 47$ )

De plus, ce besoin de reconnaissance de l'origine, de la multiplicité, et de la jouissance du féminin se retrouve dans l'association de l'être à la terre et à la mer. Cette binarité, terre et eau, est à l'origine du mythe grec des jumeaux comme Castor (fils du roi Tyndare) et Pollux (fils de Zeus, sous la forme d'un cygne). Dans Les fous de Bassan, Nora et Olivia, cousines ou plus encore «[s]œurs siamoises » (FB: 121), représentent cette gémellité. Par extension, le mythe grec transmet aussi la « croyance primitive en une âme double, une âme mortelle qui agit dans I'individu pendant sa vie, une âme immortelle qui continue sa vie dans I'au-delà. » (Zazzo, 1960 : 512) Nora, plus ancrée dans la terre et dans l'assouvissement de ces désirs d'adolescente, représente cette âme mortelle, luttant contre la patriarchie chrétienne. Olivia, quant à elle, fille de la haute mer, nostalgique et désireuse d'une fusion amoureuse, représente cette âme immortelle, figée dans l'héritage du silence et de la peur de ses ancêtres féminins.

Le limon et le cygne : nature instinctive et hermaphrodisme

Nora apparaît alors comme la « jumelle » liée à la terre. Elle est celle des deux qui se rattache aux plaisirs terrestres et qui ressent les pulsions et les désirs de son corps d'adolescente. Comme l'indique l'épigraphe de Cixous, Nora est le symbole de la force du désir féminin et d'une résistance au système patriarcal. En effet, Nora, forte de son identité féminine, se définit comme « [f]aite du limon de la terre, comme Adam, et non sortie d'entre les côtes sèches d'Adam, première comme Adam, [...] encore humide de $[s]$ a naissance unique, avide de toute connaissance terrestre et marine. » ( $F B: 116)$ Elle sait qui elle est et d'où elle vient. À travers cette conception de soi, elle s'oppose à I'histoire chrétienne dictée par les hommes de Griffin Creek. Elle refuse l'idée que la femme provienne de l'homme et, au lieu de soutenir la suprématie du masculin sur le féminin, elle établit un parallèle et un échange entre le territoire marin - domaine des femmes de Griffin Creek - et le territoire terrestre - celui des hommes de la communauté. De la même manière, elle contredit les sermons du pasteur ( $F B: 18)$ en réutilisant des paroles bibliques pour affirmer son identité: 
Et le Verbe s'est fait chair et Il a habité parmi nous. Et moi aussi, Nora Atkins, je me suis faite chair et j'habite parmi eux, mes frères et mes cousins de Griffin Creek. Le Verbe en moi est sans parole prononcée, ou écrite, réduit à un murmure secret dans mes veines. Livrée aux métamorphoses de mon âge j'ai été roulée et pétrie par une eau saumâtre, mes seins sur mes côtes viennent de se poser comme deux colombes, la promesse de dix ou douze enfants, aux yeux d'outremer, se niche dans deux petites poches, au creux de mon ventre. J'ai quinze ans. Je résonne encore de l'éclat de ma nouvelle naissance. Ève nouvelle. (FB: 118)

Le Verbe chrétien, formulé par les hommes de Griffin Creek, a empêché la reconnaissance de la femme dans sa chair et dans son corps. Nora montre qu'elle intériorise le message chrétien en ayant conscience de la naissance, mais elle exprime aussi la volupté de son corps (« roulée et pétrie »), la délicatesse de cette sensualité bourgeonnante (les seins sont comme des colombes) et l'exaltation du désir adolescent. Ce « murmure secret » de la voix patriarcale est effacé par l'éclat de sa nouvelle voix qui résonne.

De plus, tout comme Nora vient du limon, elle passe aussi son temps à modeler la glaise. Elle insiste sur le fait que, enfant, avec Olivia et Perceval, elle s'amusait à pétrir « la glaise froide et mouillée ». (FB : 115) Selon Bachelard,

cette rêverie qui naît du travail des pâtes se met aussi forcément d'accord avec une volonté de puissance spéciale, avec la joie mâle de pénétrer dans la substance, de palper l'intérieur des substances, de connaître l'intérieur des grains, de vaincre la terre intimement, comme l'eau vainc la terre, de retrouver une force élémentaire, de prendre part au combat des éléments, de participer à une force dissolvante sans recours. (Bachelard, 1942 : 146 ; l'auteur souligne)

De cette façon, Nora identifie son corps et son désir indépendamment des dictats masculins ou, plus encore, à l'égal du masculin.

Ainsi, Nora n'a pas peur de son désir et elle agit de manière audacieuse. Elle déclare que « [d]'ici la fin de l'été [elle] les [aura] tous embrassés, les uns après les autres » $(F B: 123)$ ou bien encore qu'elle sait « comment sont faits les garçons. » ( $F B: 118$ ) Elle fait le rêve d'un roi qui viendrait la chercher ou bien d'un cygne qui se transformerait en prince charmant :

[J]e me demande lequel de ces oiseaux sauvages, à la faveur de quelle obscurité profonde, se posera, un soir, sur mon toit, au cours d'un de ses voyages. Un cygne. Je suis sûre que ce sera un cygne. Il entrouvrira son plumage, je verrai son cœur à découvert qui ne bat que pour moi. $(F B: 124-125)$ 
D'après Bachelard, le cygne est un symbole hermaphrodite : « [I]e cygne est féminin dans la contemplation des eaux lumineuses ; il est masculin dans l'action. » (Bachelard, 1942 : 52) De plus, selon le mythe grec de Zeus et Léda (parents de Pollux), dans lequel Léda se métamorphose en oie pour échapper à Zeus (sous la forme d'un cygne), ce rapprochement volontaire de ces deux entités diurne et nocturne fait de cet oiseau un symbole hermaphrodite, Léda et son divin amant ne faisant qu'un. Nora est curieuse de ce rapprochement entre le masculin et le féminin ; elle alterne entre la contemplation et le jeu de séduction. Elle recherche l'union avec le masculin, de manière égale, dans le désir et la jouissance.

Cependant, les hommes de cette communauté patriarcale sont beaucoup trop agressifs dans leur désir, et les femmes beaucoup trop silencieuses dans leur désir et plaisir pour permettre à Nora d'explorer sa sexualité d'égal à égal. Elle se voudrait « chasseresse » $(F B: 126)$ mais se rend bien compte qu'elle est « trop petite encore et [que] I'ombre de l'amour qui surgit dans les conversations des mères est secrète et redoutable. » (FB: 115) Ainsi, lorsqu'elle essaie de « chasser » Stevens dans les bois, qu'il la rejette, et qu'elle fait face au « loup [qui] a la figure de [s]on oncle Nicolas » $(F B: 128)$, le lecteur comprend vite que la petite Nora ne peut pas résister au masculin. Ce loup rappelle le conte du petit chaperon rouge et la tension entre le loup prédateur et le chasseur libérateur. Dans l'analyse psychanalytique du conte par Bruno Bettelheim, le petit chaperon rouge, aux portes de la puberté, fait face à deux opposés de I'homme : le dangereux séducteur (le loup), meurtrier de la grand-mère et de la petite fille, et le chasseur, qui représente la figure paternelle, forte, responsable et qui sauve l'enfant. Dans le conte, l'enfant renaît en tant que jeune fille, libérée/éduquée par la figure paternelle, et consciente des dangers de sa naïveté (Bettelheim, 1999 : 254-277). Mais ici Nora n'est pas sauvée par le chasseur. Au contraire, sa mère lui dit toujours de faire « attention aux chasseurs » ( $F B: 126)$ et les figures paternelles la condamnent dans sa sexualité de jeune femme. L'épisode du « loup » ne se termine pas en renaissance mais plutôt avec la mort d'Irène et ensuite la mort des deux jeunes filles. Même avec ses tentatives de résistance au système patriarcal, Nora reste une victime comme ses ancêtres féminins.

La petite sirène et la marée : quête d'identité et désir de (re)connaissance

De son côté, Olivia, représente la partie « gémellaire » rattachée à l'eau et au monde de l'au-delà. Comme l'indique l'épigraphe d'Andersen, Olivia suit un parcours de vie et un destin identiques à ceux de la petite sirène. Olivia, tout d'abord entre le 
monde marin et le monde terrestre, puis dans le monde céleste, vit son désir comme une quête d'identité. Contrairement à Nora, elle a du mal à savoir qui elle est, partagée entre un héritage féminin auquel elle s'attache désespérément et un milieu familial patriarcal qui l'emprisonne. Cette confusion est visible dans la narration qui alterne entre la première personne et la troisième personne. Mais, tout comme la petite sirène, Olivia exprime une envie de se mettre en quête de son identité, avec un désir de savoir, et une recherche de sa voie et de sa voix. L'épisode qui va bouleverser sa vie est celui entre Stevens et elle, enfants, jouant dans le sable à la plage. Après cet épisode et celui de la punition corporelle du père de Stevens dont elle est témoin, Olivia comprend la menace du système patriarcal et adopte la posture silencieuse des femmes de Griffin Creek. Selon Andrea King, « [m]ale violence triggers Olivia's retreat into the protective realm of the feminine, but the above passage also demonstrates her ambivalence with regard to fleeing Stevens and Griffin Creek. ${ }^{5}$ » (King, 2012 : 34) En effet, l'ambiguïté du personnage réside, comme dans le personnage de la petite sirène, dans la problématique du « dedans-dehors ». Olivia voudrait partir de Griffin Creek, mais elle est retenue là par son désir de comprendre les hommes aussi bien que les femmes de cette communauté.

Olivia, tout d'abord, ne sait pas comment exprimer son désir et peine à comprendre sa sexualité féminine car sa mère est morte quand elle était jeune. Elle recherche constamment, dans l'eau, l'image de sa mère : «Cette frange d'écume à ses pieds est-ce la robe de sa mère ? [...] Si je regarde bien, [...] [I] mystère de la vie et de la mort de ma mère n'aura plus de secret pour moi. » (FB: 211) Le mystère de vie et de mort peut être compris comme le mystère de l'acte sexuel et du plaisir. Sa mère est une présence distante, souffrant d'un chagrin secret, victime d'une « jeunesse tuée » (FB: 208), avant de disparaître. Les seuls gestes féminins qu'Olivia connaisse sont les travaux ménagers auxquels elle s'adonne mécaniquement en silence :

Prendre la température du fer en l'approchant de sa joue doucement. Ainsi faisaient sa mère et sa grand-mère. La longue lignée des gestes de femme à Griffin Creek pour la lier à jamais.

Et le vent qui tourbillonne tout autour de la maison fait résonner Griffin Creek avec des voix de femmes patientes, repasseuses, laveuses, cuisinières, épouses, grossissantes, enfantantes, mères des vivants et des morts, désirantes et désirées dans le vent amer. (FB: 215)

5. Ma traduction : Cette violence masculine pousse Olivia à se retirer dans le domaine protecteur du féminin, mais le passage ci-dessus montre son ambivalence à quitter Stevens et Griffin Creek. 
Il y a donc un problème du système patriarcal qui condamne les femmes à rester dans un espace physique et moral de restriction et de simplicité. En dépit de leurs désirs, elles restent silencieuses et patientes. Selon Isabelle Boisclair, la principale réponse à la domination masculine viendrait de la solidarité féminine. Le problème est que ce soutien se transforme en transmission de I'horreur et de la peur. À cause de cela, les voix fantômes des femmes de la communauté ne font que maintenir Olivia « dans l'ordre patriarcal plutôt que de constituer des symboles d'affranchissement. » (Boisclair, 2008 : 29)

Ainsi, ce qui retient le fantôme d'Olivia à Griffin Creek, ce n'est pas vraiment un désir inassouvi, mais plutôt le besoin de comprendre ce désir : « Non, non ce n'est pas moi, c'est le désir qui me tire et m'amène, chaque jour, sur la grève. J'en demande pardon aux grandes femmes liquides, mes mère et grand-mères. » (FB:221) Même si Olivia a peur du masculin parce que l'héritage féminin la pousse à cela, elle aimerait aussi connaître la vérité sur le rapport homme-femme :

Si seulement je voulais bien j'apprendrais tout de lui, d'un seul coup, la vie, la mort, tout. Je ne serais plus jamais une innocente simplette qui repasse des chemises en silence. L'amour seul pourrait faire que je devienne femme à part entière et communique d'égale à égale avec mes mère et grand-mères, dans l'ombre et le vent, à mots couverts, d'un air entendu, du mystère qui me ravage, corps et âme. ( $F B: 216)$

Ce n'est pas seulement le système patriarcal qui a condamné Olivia dans son désir et sa sexualité féminine, ce sont aussi le silence des femmes et l'absence d'amour et de sens dans leurs voix.

Anne Hébert, dans Les fous de Bassan, dépeint une société qui condamne aussi bien les femmes que les hommes. La frustration des hommes, la peur des femmes, et surtout le manque de communication et de reconnaissance du désir entre le masculin et le féminin bloquent cette communauté dans un système où personne ne gagne. Le récit semble donner l'indication d'une solution potentielle à travers le personnage de Perceval. Jeune homme naïf, entre deux âges, entre terre et mer, entre Stevens/ Nicolas et Nora/Olivia, Perceval représente le cri, « la voix primaire de l'idiot. » ( $F B: 232$ ) Reprenant les paroles de Lacan, Jean Abitbol explique que « le cri n'est pas d'abord appel, mais il fait surgir le silence. » (Abitbol, 2005 : 363) Ainsi, Perceval ferait exploser le silence des femmes et des hommes afin de représenter le besoin de l'expression des sexualités et la nécessité d'identités sexuelles plus ouvertes. 


\section{Bibliographie}

ABITBOL, Jean (2005), L'odyssée de la voix, Paris, Éditions Robert Laffont.

ANZALDÚA, Gloria (1987), Borderlands, La Frontera, San Francisco, Aunt Lute Books.

BACHELARD, Gaston (1942), L'eau et les rêves, Paris, Éditions José Corti.

BETTELHEIM, Bruno (1999 [1976]), Psychanalyse des contes de fées, Paris, Éditions Pocket.

BOISCLAIR, Isabelle (2008), " La solidarité féminine comme réponse à la domination masculine : étude de deux motifs genrés dans l'œuvre d'Anne Hébert », Les Cahiers Anne Hébert, n 8 : 15-36.

BROCHU, André (2000), Anne Hébert, le secret de vie et de mort, Ottawa, Les Presses de l'Université d'Ottawa.

CIXOUS, Hélène (1975), « Le rire de la Méduse », L’Arc, n 61 : 39-54.

FISHER, Claudine (2001), «Féminitude et folie dans Les fous de Bassan d'Anne Hébert », dans Janis. L Pallister (dir), The Art and Genius of Anne Hébert, Essays on Her Works. Night and the Day Are One, Madison et Teaneck, Fairleigh Dickinson University Press / London, Associates University Presses : 63 -74.

FREUD, Sigmund (1974 [1953]), "The Medusa's Head », dans The Standard Edition of the Complete Psychological Works of Sigmund Freud, vol. 18, trad. James Strachey [et al.], London, Hogarth Press.

HÉBERT, Anne (1982), Les fous de Bassan, Paris, Seuil.

JONES, Ann Rosalind (2000), " "Blond chef, grande conqueste" : Feminist Theories of the Gaze, the blason anatomique, and Louise Labé's Sonnet 6 », dans John O'Brien et Malcolm Quainton (dir.), Distant Voices Still Heard : Contemporary Readings of French Renaissance Literature, Liverpool, Liverpool University Press : 85-106.

KING, Andrea (2012), « Haunting Love in Anne Hébert's Les fous de Bassan and Mary Novik's Conceit », Canadian Literature, $\mathrm{n}^{\circ} 214: 31-45$.

LETENDRE, Evelyne (2007), Figures de l'homme en prédateur: modèles et contre-modèles dans quatre romans québécois écrits par des femmes depuis 1980, mémoire de maîtrise, Université de Sherbrooke.

MCDOUGALL, Joyce (1995), Many Faces of Eros : A Psychoanalytic Exploration of Human Sexuality, New York, Norton.

ZAZZO, René (1960), Les jumeaux, le couple et la personne, tome II, Paris, Presses universitaires de France. 